\title{
Komplementärmedizin in Exeter: Ein 10-jähriges Jubiläum
}

Im Jahre 1993 ermöglichte die Maurice Laing Foundation den Aufbau einer Forschungsgruppe zur Komplementärmedizin an der Universität Exeter, Grossbritannien. Die in ihren Anfängen noch kleine Forschungsgruppe hatte sich zum Ziel gesetzt, die Sicherheit und Effektivität von Behandlungsweisen in der Komplementärmedizin zu erforschen. Letztlich ging es dieser Gruppe darum, den Nutzen dieser Behandlungsweisen $\mathrm{zu}$ maximieren und Risiken für Patienten $\mathrm{zu}$ minimieren. Unter der Forderung einer rigorosen, interdisziplinären und internationalen Forschung sollte das analytische Denken auf dem Gebiet der Komplementärmedizin gefördert werden.

In den vergangenen 10 Jahren folgte ein kontinuierlicher Aufbau der Forschungsgruppe. Forschungsschwerpunkte sind heute das Erstellen von systematischen Reviews und die Durchführung von klinischen Studien. Derzeit arbeiten in der Abteilung 13 feste Mitarbeiter und mehrere Gastwissenschaftler aus aller Welt - einer aus Japan, zwei aus Sri Lanka und zwei weitere aus Korea. Die mittlerweile zirka 600 Veröffentlichungen in verschiedenen Fachzeitschriften sowie die mehr als ein Dutzend Buchveröffentlichungen zum Thema Komplementärmedizin sind Zeugnis der umfassenden wissenschaftlichen Arbeit der letzten Jahre. Der «Desktop Guide to Complementary and Alternative Medicine - an EvidenceBased Approach» [1] sollte hervorgehoben werden. Er beinhaltet eine umfassende Evidenz-basierende Übersicht zur Komplementärmedizin und wurde in führenden Fachblättern wie JAMA, Lancet oder Annals of Internal Medicine über alle Massen gelobt.

Als die Abteilung im letzten Jahr als weltweit führendes Forschungszentrum zur Erforschung der Komplementärmedizin genannt wurde [2], haben dies die Mitarbeiter als grosses Kompliment und Anerkennung ihrer Arbeit aufgefasst. Eine komplette Liste aller Publikationen ist auf Anfrage unentgeltlich erhältlich (E-mail: Edzard.Ernst@pms.ac.uk).

Die nähere Zukunft der Abteilung sieht rosig aus: Kürzlich erneuerte die Maurice Laing Foundation ihre finanzielle Unterstützung. Bedeutend ist auch, dass die Abteilung für Komplementärmedizin vor etwa einem Jahr an die Peninsula Medical School der Universitäten von Exeter und Plymouth angeschlossen wurde. Dies wird aller Voraussicht nach eine intensive Beteiligung an der medizinischen Lehre und eine Ausweitung der Aktivitäten auf die Grundlagenforschung mit sich bringen.

Die Abteilung wird ihr 10-jähriges Jubiläum angemessen feiern. Der Geburtstag soll anlässlich des «10th Annual Symposium on Complementary Health Care» zelebriert werden. Diese Tagung hat sich inzwischen zu einem wichtigen internationalen Ereignis ausgeweitet. Sie wird heuer dem Anlass entsprechend in dem «Royal College of Physicians» in London stattfinden (21., 22. November 2003). Weitere Details: http:// www.ex.ac.uk/FACT/sympo.htm.

K. Schmidt, Exeter

\section{Literatur}

1 Ernst E, Pittler MH, Stevinson C, White AR, Eisenberg D: The Desktop Guide to Complementary and Alternative Medicine. Edinburgh, Mosby, 2001.

2 Hentschel C: Profiling 'centres of excellence' in CAM research. Complement Ther Med 2002;10:46-48

\begin{tabular}{|c|c|c|}
\hline KARGER & (c) 2003 S. Karger GmbH, Freiburg & $\begin{array}{l}\text { Katja Schmidt } \\
\text { Complementary Medicine, Peninsula Medical School }\end{array}$ \\
\hline $\begin{array}{l}\text { Fax }+497614520714 \\
\text { E-mail Information@Karger.de } \\
\text { www.karger.com }\end{array}$ & $\begin{array}{l}\text { Accessible online at: } \\
\text { www.karger.com/fkm }\end{array}$ & $\begin{array}{l}\text { Universities of Exeter and Plymouth, Institute of Health and Social Care } \\
25 \text { Victoria Park Road } \\
\text { GB-Exeter EX2 4NT } \\
\text { E-mail katja.schmidt@pms.ac.uk }\end{array}$ \\
\hline
\end{tabular}

